\title{
DEHOMOGENIZATION OF GRADINGS TO ZARISKIAN FILTRATIONS AND APPLICATIONS TO INVERTIBLE IDEALS
}

\author{
HUI-SHI LI AND FREDDY VAN OYSTAEYEN
}

(Communicated by Maurice Auslander)

\begin{abstract}
The method of dehomogenizing graded rings has been used successfully in algebraic geometry, e.g., a determinental ring is a dehomogenization of a Schubert cycle. We extend this method to noncommutative graded rings, dehomogenizing suitably graded rings to Zariski filtered rings and deriving, in a very elementary way, homological properties related to Auslander regularity and the Gorenstein property for noncommutative rings. As an application we study the lifting of such properties from a quotient modulo an invertible ideal.
\end{abstract}

\section{INTRODUCTION}

In projective algebraic geometry, homogeneous coordinate rings appear together with a suitable dehomogenization. For example, if $V(I)$ is a projective variety determined by a homogeneous ideal $I$ of the polynomial ring $k\left[X_{0}, \ldots, X_{n}\right]$ and $R$ is the graded coordinate ring $k\left[X_{0}, \ldots, X_{n}\right] / I$, then $A=R /\left(1-\bar{X}_{0}\right) R$ is a isomorphic to the coordinate ring of the open affine subvariety complementary to the hyperplane "at infinity" (defined by the vanishing of $X_{0}$ ) in $V(I)$. In a similar way every determinantal ring is a dehomogenization of a Schubert cycle (being the graded coordinate ring of a Schubert variety), and this dehomogenization principle is the basis for the study of determinantal rings (cf. [BV] for detail). We now extend a similar method to certain graded rings such that a suitable dehomogenization is a Zariskian filtered ring having the original graded ring as its Rees ring in the sense of [1, 10]. For example, the $(2 n+1)$-dimensional Heisenberg Lie algebra $\mathbf{g}$ has an enveloping algebra $U(\mathbf{g})$, which turns out to be the Rees ring of the $n$th Weyl algebra $A_{n}(k)$, assuming char $k=0$, filtered by the $\Sigma$-filtration (this is not the Bernstein filtration!). One of the immediate corollaries of this viewpoint is: gl. $\operatorname{dim}(U(\mathbf{g}))=G K \cdot \operatorname{dim}(U(\mathbf{g}))=2 n+1$. We study in some detail Noetherian properties, Auslander regularity properties, and Gorenstein properties. This provides a remarkable reversion of methods; in [17, 10, 12, 9] Zariskian filtrations have been investigated by using graded techniques on graded modules over the Rees ring and the associated graded ring of the filtration, but now we use the results on filtered rings in deriving results for graded rings. Moreover,

Received by the editors March 29, 1990 and, in revised form, November 20, 1990.

1980 Mathematics Subject Classification (1985 Revision). Primary 16A03, 16A50.

The first author was supported by a grant of Antwerp province. 
the generalized Rees ring used in [4, 5] turns out to be a Rees ring of a suitable filtration, and this allows us to lift certain ungraded properties modulo invertible ideals. In fact we can close a double loop and establish graded versions of the latter ungraded results (under weaker graded conditions) that have some independent interest.

\section{Preliminaries}

Let $A$ be a filtered ring with filtration $F A=\left\{F_{n} A, n \in \mathbb{Z}\right\}$. Then there are two graded rings determined by $F A$, i.e., the associated graded ring $G(A)=$ $\bigoplus_{n \in \mathbb{Z}} F_{n} A / F_{n-1} A$ and the Rees ring $\tilde{A}=\bigoplus_{n \in \mathbb{Z}} F_{n} A$. Similarly, for any filtered $A$-module $M$ with filtration $F M=\left\{F_{n} M, n \in \mathbb{Z}\right\}$, one defines the associated graded module of $M: G(M)=\bigoplus_{n \in \mathbb{Z}} F_{n} M / F_{n-1} M$ and the associated Rees module of $M: \widetilde{M}=\bigoplus_{n \in \mathbb{Z}} F_{n} M$. Consequently, there are two associated functors from the category of filtered (left) $A$-modules $A$-filt to the category of graded (left) $G(A)$-modules $G(A)$-gr and the category of graded (left) $\tilde{A}$ modules $\widetilde{A}$-gr respectively: $G(): M \mapsto G(M), \widetilde{()}: M \mapsto \widetilde{M}$. Let $X$ denote the image of $1 \in F_{1} A$ in $\tilde{A_{1}}$. Then it is central regular in $\tilde{A}$. The class of $X$-torsionfree graded $\tilde{A}$-modules, i.e., the graded modules in which there is no nonzero element annihilated by $X$, forms a full subcategory of $\tilde{A}$-gr, denoted by $\mathscr{F}_{X}$. The following lemma sums up various relations between the filtered and associated graded objects.

2.1. Lemma [1]. Let $A$ be a filtered ring with filtration $F A$ and $M \in A$-filt with filtration $F M$. Put $I=X \tilde{A}$.

(1) $\widetilde{A} / I \cong G(A), \widetilde{M} / I M \cong G(M)$.

(2) $\left.\widetilde{A} /(1-X) \widetilde{A} \cong A, M \cong \widetilde{M} /(1-X) \widetilde{M}=\lim _{(\widetilde{M}}\right)_{n}$, where the maps in the inductive system are given by the multiplication of $X$ and there are isomorphisms of additive groups: $F_{n} M \cong(\widetilde{M})_{n}+(1-X) \widetilde{M} /(1-X) \widetilde{M}, n \in \mathbb{Z}$.

(3) The functor $\widetilde{()}$ defined above determines an equivalence of categories between $A$-filt and $\mathscr{F}_{X}$.

(4) The localization of $\tilde{A}$ at the multiplicatively closed set $\left\{1, X, X^{2}, \ldots\right\}$, denoted by $\widetilde{A}_{(X)}$, equals $A\left[X, X^{-1}\right]$. Also, $\widetilde{M}_{(X)}=M\left[X, X^{-1}\right]$.

The foregoing lemma is the basis for many results on filtered rings, in particular for the so-called Zariskian filtered rings including both positively filtered rings (Weyl algebra and the enveloping algebra of a finite-dimensional Lie algebra) and the filtered rings with nontrivial negative part (classical Zariski rings with $I$-adic filtrations and the ring of microlocal differential operators), e.g., [1, $17,8-12,6]$, etc.

From Lemma 2.1 it is clear that any filtered ring is a dehomogenization of its associated Rees ring. In this note we do the opposite, i.e., we show that by taking a suitable dehomogenization, many of the graded rings may be made into Rees rings associated to Zariskian filtered rings. So in this way one also gets information for a given graded ring from its associated filtered ring.

\section{Dehomogenization}

Let $R=\bigoplus_{n \in \mathbb{Z}} R_{n}$ be any $\mathbb{Z}$-graded ring and $X$ a homogeneous element of degree 1 in $R$. Then since for any homogeneous element $r_{n} \in R_{n}$ and $t>0$, 
we have $r_{n}=X^{t} r_{n}+\left(1-X^{t}\right) r_{n}$ and the quotient ring $A=R /\langle 1-X\rangle$ where $\langle 1-X\rangle$ is the ideal of $R$ generated by $1-X$ may be made into a filtered ring by endowing it with the filtration

$$
F A=\left\{F_{n} A=\left(R_{n}+\langle 1-X\rangle\right) /\langle 1-X\rangle, n \in \mathbb{Z}\right\} .
$$

Obviously the filtration $F A$ defined on $A$ is exhaustive.

3.1. Lemma. With the above notations, if $X$ is a regular homogeneous element, then

(1) $(1-X) R \cap R_{n}=0$ for all $n \in \mathbb{Z}$.

(2) If $X$ is also a normal element (i.e., $X R=R X$ ) then $X$ is central if and only if $(1-X) R$ is an ideal of $R$.

Proof. (1) is straightforward. To prove (2), let us consider the natural morphism $\pi: R \rightarrow R /(1-X) R$. Since $R$ is graded and $X$ is normal, if we look at the image of $X r=r^{\prime} X$ under the morphism $\pi$ for any homogeneous element $r$ in $R$, then the property (1) yields the equivalence immediately.

Recall from [NVO] that for a $G$-graded ring $R$ by a group $G$, the graded Jacobson radical of $R$, denoted by $J^{g}(R)$, is the largest proper graded ideal of $R$ such that for all $a \in J^{g}(R) \cap R_{e}$ it follows that $1+a r$ is a unit. In other words, $J^{g}(R)$ is the largest proper graded ideal of $R$ such that its intersection with $R_{e}$ is contained in the Jacobson radical of $R_{e}$ where $e$ is the neutral element of $G$.

3.2. Proposition. Let $R$ be a $\mathbb{Z}$-graded ring and $X$ a regular central homogeneous element of degree 1 in $R$. With the above notations,

(1) $G(A) \cong R / X R$ as graded rings.

(2) $\tilde{A} \cong R$ as graded rings.

(3) $X \in J^{g}(R)$ if and only if $F_{-1} A \subset J\left(F_{0} A\right)$, where $J\left(F_{0} A\right)$ denotes the Jacobson radical of $F_{0} A$.

(4) The localization of $R$ at the Ore set $\left\{1, X, X^{2}, \ldots\right\}$ exists; it is denoted by $R_{(X)}$, and the natural homomorphism $\pi: R \rightarrow A$ factors through $R_{(X)}$ in a canonical way, so there is a commutative diagram

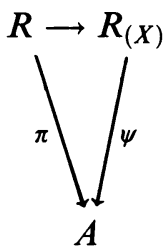

where $R_{(X)}$ is still a graded ring, i.e., $R_{(X)}=\bigoplus_{n \in \mathbb{Z}}\left[R_{(X)}\right]_{n},\left[R_{(X)}\right]_{n}=\left\{X^{j} r ; j \in\right.$ $\left.\mathbb{Z}, \quad r \in R_{n-j}\right\}$, and $\psi\left(X^{j} r\right)=r+(1-X)$. Note that $R_{(X)}$ is a strongly graded ring and its structure is particularly simple (see [NVO, Chapter 1]), i.e., $\left[R_{(X)}\right]_{0}\left[x, x^{-1}\right] \cong R_{(X)}$ where $x$ corresponds to $X$.

(5) With notation as in (4), the homomorphism $\psi$ maps $\left[R_{(X)}\right]_{0}$ isomorphically onto $A$.

Proof. By Lemma 3.1 and results of [NVO], (2), (3), and (5) may be verified easily, so we only include the proof of (1) and (3) here. 
Since, by definition,

$$
G(A)=\bigoplus_{n \in \mathbb{Z}} \frac{R_{n}+(1-X) R}{R_{n-1}+(1-X) R}, \quad R / X R=\bigoplus_{n \in \mathbb{Z}} R_{n}+X R / X R .
$$

For each $n$ one may define an isomorphism of additive groups $\varphi_{n}: G(A)_{n} \rightarrow$ $R_{n}+X R / X R$ as follows: $r_{n}+R_{n-1}+(1-X) R \mapsto r_{n}+X R$. Indeed, since for every $r_{n-1} \in R_{n-1}$ we have $r_{n-1}=X r_{n-1}+(1-X) r_{n-1}$, it follows that $\varphi_{n}$ is well defined. Moreover, if $r_{n} \in X R$ then $r_{n}=X r_{n-1}$ for some $r_{n-1} \in R_{n-1}$. Hence $r_{n}=X r_{n-1}=r_{n-1}-(1-X) r_{n-1}$. This shows that $\varphi_{n}$ is an isomorphism. If we combine all $\varphi_{n}$, then the required graded ring isomorphism is obtained.

Suppose $X \in J^{g}(R)$. It follows that $X R_{-1} \subset J^{g}(R) \cap R_{0}$, i.e., $1-X r_{-1}$ is invertible in $R_{0}$ for every $r_{-1} \in R_{-1}$. But then it follows from $r_{-1}=$ $X r_{-1}+(1-X) r_{-1}, r_{-1} \in R_{-1}$, that $R_{-1}+(1-X) R /(1-X) R=F_{-1} A \subset J\left(F_{0} A\right)$. The converse follows from Lemma 3.1 (1).

Example (i). Let us look at the $n$th Weyl algebra $A_{n}(k)$ over a field $k$ of characteristic zero. It is well known that $A_{n}(k) \cong U(\mathbf{g}) /(1-z) U(\mathbf{g})$, where $U(\mathbf{g})$ is the enveloping algebra of the $(2 n+1)$-dimensional Heisenberg Lie algebra with basis $\left\{x_{1}, \ldots, x_{n}, y_{1}, \ldots, y_{n}, z\right\}$ over $k$. We claim that $U(\mathbf{g})$ is the Rees algebra of $A_{n}(k)$ with respect to the $\Sigma$-filtration on $A_{n}(k)$ (see [2]), i.e., $\sum_{n}=\left\{Q=\sum_{\alpha \leq n} q_{\alpha}(x) y^{\alpha}\right\}$, the set of differential operators of order $\leq n$ where $q_{\alpha}(x) \in k\left[x_{1}, \ldots, x_{n}\right]$. Indeed, if we put $\operatorname{deg}\left(x_{i}\right)=0, \operatorname{deg}\left(y_{i}\right)=1$ for $i=1, \ldots, n$ in the tensor algebra $T(\mathbf{g})$ determined by $\mathbf{g}$, then $U(\mathbf{g})$ becomes a graded algebra with $z$ a central regular homogeneous element of degree 1 , and the filtration we defined on $U(\mathbf{g}) /(1-z) U(\mathbf{g})$ as before is actually the $\Sigma$ filtration. Hence $\widetilde{A_{n}(k)} \cong U(\mathbf{g})$ by Proposition 3.2. Since the associated graded rings of $A_{n}(k)$ with respect to both the standard-and the $\Sigma$-filtration are the same, i.e., the polynomial ring in $2 n$ variables over $k$, it follows from [9] that gl. $\operatorname{dim}(U(\mathbf{g}))=2 n+1=\mathrm{GK} \cdot \operatorname{dim}(U(\mathbf{g}))$ (the latter one is the Gelfand-Kirillov dimension).

Example (ii). Let $R$ be any $\mathbb{Z}$-graded ring. Consider the polynomial ring $R[T]$ over $R$ in $T$ and the "mixed" gradation on $R[T]$,

$$
R[T]_{n}=\left\{\sum_{i+j=n} r_{i} T^{j}, r_{i} \in R_{i}\right\}, \quad n \in \mathbb{Z} .
$$

Then

(1) $T \in R[T]_{1}$, and this gradation is still positive or left limited if the original one is.

(2) The filtered ring $A=R[T] /(1-T) R[T]$ with filtration $F A$ determined by the "mixed" gradation has associated graded ring $G(A) \cong R[T] / T R[T] \cong R$ as graded rings and Rees ring $\tilde{A} \cong R[T]$ as graded rings. But on the other hand, $A$ is obviously isomorphic to $R$ as an ungraded ring.

Note that $R$ is a graded subring of $R[T]$. In view of Lemma 2.1 there are nice relations between the categories $A$-filt, $R$-gr, and $R[T]$-gr. For example, if we take an $R$-module $M$ and filter it, then there is an exact sequence

$$
0 \rightarrow \widetilde{M} \rightarrow \widetilde{M} \rightarrow M \rightarrow 0
$$


of $R[T]$-modules, but this is also an exact sequence of $R$-modules and $\widetilde{M}$ is in $R$-gr. Since p. $\operatorname{dim}_{R} \widetilde{M}=$ gr.p. $\operatorname{dim}_{R} \widetilde{M}$ (see [NVO14]), it follows immediately that $\operatorname{gl} . \operatorname{dim} R \leq 1+$ gr. gl. $\operatorname{dim} R$, where gr.p.dim resp. gr.gl. dim represents the graded projective dimension of $\widetilde{M}$ resp. the graded global dimension of $R$ (compare with [NVO14] $P_{120}$ ). Also note that a ring $R$ has finite injective dimension (i.e., every $R$-module has a finite injective resolution) if and only if there exists $k \geq 0$ such that $\operatorname{Ext}_{R}^{j}(M, R)=0$ for all $R$-modules $M$ and $j>k$. Similarly, we obtain $\operatorname{inj} . \operatorname{dim} R \leq 1+$ gr. inj. $\operatorname{dim} R$.

Another use of (1) and (2) above will be given later.

Recall from [10] that a filtered ring $A$ with filtration $F A$ is called a left Zariskian filtered ring (or $F A$ is called a Zariskian filtration) if the associated Rees ring $\tilde{A}$ of $A$ is left Noetherian and $F_{-1} A$ is contained in the Jacobson radical $J\left(F_{0} A\right)$ of $F_{0} A$. There are at least eight different characterizations of a Zariskian filtered ring; we recall one of them here and refer the reader to [6, 11] for more details.

3.3. Theorem. A filtered ring $A$ with filtration $F A$ is left Zariskian if and only if $G(A)$ is left Noetherian and the completion $\widehat{A}$ of $A$ with respect to $F A$ is a faithful flat right $A$-module.

Let us consider a generalization of the Hilbert basis theorem to graded rings.

3.4. Theorem. Let $R$ be a $\mathbb{Z}$-graded ring and $X$ a regular central homogeneous element of degree 1 in $R$. Then the following statements are equivalent:

(1) $R$ is a left Noetherian ring, and $X \in J^{g}(R)$.

(2) $R / X R$ is left Noetherian, and the completion $\widehat{A}$ of $A(=R /(1-X) R)$ with respect to the filtration $F A$ is a faithful flat right $A$-module (or equivalently, $A$ is a left Zariskian filtered ring).

Proof. The proof follows from the definition of Zariskian filtration and Proposition 3.2(3).

When the given graded ring $R$ has a positive or left limited gradation, i.e., there exists an integer $c$ such that $R_{n}=0$ for all $n<c$, Theorem 3.4 may be reduced to

3.5. Theorem. Let $R$ have a positive or left limited gradation. Let $X$ be a regular central homogeneous element of degree 1 in $R$. The following statements are equivalent:

(1) $R$ is left Noetherian.

(2) $R / X R$ is left Noetherian.

Proof. Note that under the assumption we always have $\widehat{A}=A$ or in other words $A$ is complete with respect to its filtration $F A$. Hence by Proposition 3.2(1) and Theorem 3.3, $A$ is Zariskian, and consequently $\tilde{A}$ is Noetherian. But by Proposition $3.2(2) R$ is certainly Noetherian too. The implication (1) $\Rightarrow(2)$ is trivial.

Let $R$ be any $\mathbb{Z}$-graded ring and $I$ a graded ideal of $R$ with a centralizing sequence of homogeneous generators $\left\{X_{1}, \ldots, X_{n}\right\}$ of degree 1 , i.e., for each $j \in\{0, \ldots, n-1\}$ the image $\bar{X}_{j+1}$ of $X_{j+1}$ in $\bar{R}_{j}=R / \sum_{i}^{j} X_{i} R$ is central element. We introduce two conditions on the ideal $I$ :

(RN) $\bar{X}_{j+1}$ is regular in $\bar{R}_{j}$. 
(JN) $\bar{X}_{j+1} \in J^{g}\left(\bar{R}_{j}\right)$.

Now an easy induction yields the following

3.6. Corollary. Let $R$ be a $\mathbb{Z}$-graded ring and $I$ an ideal of $R$ with a centralizing sequence of homogeneous generators $\left\{X_{1}, \ldots, X_{n}\right\}$ of degree 1 . Suppose that I satisfies the condition (RN). Then the following are equivalent:

(1) $R$ is left Noetherian, and I satisfies the condition (JN).

(2) For each $j \in\{0, \ldots, n-1\}$ the ring $\bar{R}_{j} /\left(1-\bar{X}_{j+1}\right) \bar{R}_{j}$ is a left Zariskian filtered ring with the defined filtration where $\bar{R}_{j}=R / \sum_{i}^{j} X_{i} R$.

3.7. Corollary. Let $R$ have a positive or left limited $\mathbb{Z}$-gradation and $I$ an ideal of $R$ with a centralizing sequence of homogeneous generators $\left\{X_{1}, \ldots, X_{n}\right\}$ of degree 1. Suppose that I satisfies the condition (RN). Then the following are equivalent:

(1) $R$ is left Noetherian.

(2) $R / I$ is left Noetherian.

\section{LIFTING PROPERTIES}

First recall

4.1. Theorem [17]. Let $R$ be a $\mathbb{Z}$-graded Noetherian ring, and let $X$ be a central regular homogeneous element of positive contained in $J^{g}(R)$. If $R / X R$ is a gr-maximal order in a gr-simple gr-Artinian ring, then $R$ is a gr-maximal order in a gr-simple gr-Artinian ring.

4.2. Proposition. Let $R$ be a $\mathbb{Z}$-graded Noetherian ring and $I$ an ideal of $R$ with a centralizing sequence of homogeneous generators $\left\{X_{1}, \ldots, X_{n}\right\}$ of degree 1. Suppose that I satisfies the conditions (RN) and (JN). If $R / I$ is a gr-maximal order in a gr-simple gr-Artinian ring, then $R$ resp. $R /\left(1-X_{1}\right) R$ is a gr-maximal resp. maximal order in a gr-simple gr-Artinian resp. simple Artinian ring.

Proof. We will only prove the theorem for $n=1$. (For $n>1$ an easy induction may be used.) By our assumptions the filtered ring $A=R /\left(1-X_{1}\right) R$ with filtration as before is Zariskian (Theorem 3.4). Hence our assertions follow from Proposition 3.2 and [17, Theorem 5].

4.3. Proposition. Let $R$ have a positive or left limited gradation and $I$ an ideal of $R$ with a normalizing sequence of homogeneous generators $\left\{X_{1}, \ldots, X_{n}\right\}$ of degree 1. Suppose that I satisfies the condition (RN). If $R / I$ is a Noetherian gr-maximal order in a gr-simple gr-Artinian ring, then $R$ resp. $R /\left(1-X_{1}\right) R$ is a Noetherian gr-maximal resp. maximal order in a gr-simple gr-Artinian resp. simple Artinian ring.

Proof. For $n=1$ consider the filtered ring $R /\left(1-X_{1}\right) R$ as before. Then this follows from Proposition 3.2, Theorem 3.5, and [17, Theorem 5]. An induction completes the proof for $n>1$.

Finally, recall from homological algebra (see $[15,16])$ that if $R$ is a Noetherian ring and $M$ is a finitely generated $R$-module with finite projective dimension, then there exists a unique smallest integer $j_{R}(M)$ such that $\operatorname{Ext}_{R}^{j_{R}(M)}(M, R)$ $\neq 0$. The number $j_{R}(M)$ is called the grade number of $M$. 
A (Z-graded) ring $R$ is called (graded) Gorenstein resp. Auslander regular if (A1) $R$ is left and right Noetherian.

(A2) inj. $\operatorname{dim} R=\mu<\infty$ resp. gl. $\operatorname{dim} R=\mu<\infty$ where inj. $\operatorname{dim} R$ denotes the injective dimension of $R$.

(A3) For any nonzero finitely generated (graded) left and right $R$-module $M$, any $0 \leq k \leq \mu$, and any nonzero (graded) submodule $N \subset \operatorname{Ext}_{R}^{k}(M, R)$, it follows that $\operatorname{Ext}_{R}^{l}(N, R)=0$ for all $l<k$.

4.4. Theorem [7]. Let $R$ be a left and right Noetherian ring and $X$ a regular central element of $R$. Let $R_{(X)}$ denote the localization of $R$ at the Ore set $\left\{1, X, X^{2}, \ldots\right\}$. Suppose that $R / X R$ and $R_{(X)}$ are Auslander regular. Then $R$ is Auslander regular.

4.5. Theorem. Let $R$ have a positive or left limited gradation and $I$ an ideal of $R$ with a centralizing sequence of homogeneous generators $\left\{X_{1}, \ldots, X_{n}\right\}$ of degree 1 . Suppose that I satisfies the condition (RN). If $R / I$ is graded Auslander regular, then $R$ is Auslander regular.

Proof. As before we only prove the case of $n=1$. By Theorem $3.5 R$ is left and right Noetherian. Consider the filtered ring $A=R /\left(1-X_{1}\right) R$ with filtration $F A$. Then it is Zariskian (Theorem 3.4). It follows from [10] that $A$ is Auslander regular since $G(A) \cong R / X_{1} R$. But by Proposition 3.2 we know that $A\left[x, x^{-1}\right] \cong R_{\left(X_{1}\right)}$. It follows that $R_{\left(X_{1}\right)}$ is Auslander regular. Finally, Theorem 4.4 entails that $R$ is Auslander regular.

2.6. Proposition. Let $R$ have a positive or left limited $\mathbb{Z}$-gradation. If $R$ is graded Auslander regular, then it is Auslander regular.

Proof. Consider the polynomial ring $R[T]$ over $R$ in $T$ and the "mixed" gradation on $R[T]$ as in Example (ii) of $\S 3$. Then $T \in R[T]_{1}$, and this gradation is still positive or left limited. It follows that the filtered ring $A=$ $R[T] /(1-T) R[T]$ with filtration $F A$ determined by the "mixed" gradation is Zariskian since $G(A) \cong R[T] / T R[T] \cong R$ as graded rings. Hence by [10] $A$ is Auslander regular. But since $A$ is also isomorphic to $R$ as an ungraded ring, $R$ is Auslander regular, too.

4.7. Corollary. Let $R$ have a positive or left limited $\mathbb{Z}$-gradation and $I$ an ideal of $R$ with a centralizing sequence of homogeneous generators $\left\{X_{1}, \ldots, X_{n}\right\}$ that are not necessarily of degree 1 . Suppose that I satisfies the conditions (RN) and (JN). If $R / I$ is Auslander regular, then $R$ is Auslander regular.

Proof. As before we only give the proof for $n=1$. Since $X_{1} \in J^{g}(R)$, it follows from [9, Proposition 5.3] that $R$ is graded Auslander regular. Hence by Proposition $4.6 R$ is Auslander regular.

When the given gradation is not left limited we have the following

4.8. Proposition. Let $R$ be a left and right Noetherian $\mathbb{Z}$-graded ring and $I$ an ideal of $R$ with a centralizing sequence of homogeneous generators $\left\{X_{1}, \ldots, X_{n}\right\}$ of degree 1. Suppose that $I$ also satisfies the conditions $(\mathrm{RN})$ and $(\mathrm{JN})$. If $R / I$ is graded Auslander regular then $R$ is Auslander regular.

Proof. For $n=1$ consider the filtered ring $A=R /\left(1-X_{1}\right) R$ with filtration $F A$; then it is Zariskian by Theorem 3.4. It follows from [10] that $A$ is 
Auslander regular since $G(A) \cong R / X_{1} R$ by Proposition 3.2. Now a similar argument as in the proof of Proposition 4.5 finishes the proof.

4.9. Remark. When $n=1$, Corollary 4.7 and Proposition 4.8 are special cases of [9, Theorem 5.9], but the proofs we gave here are very simple. In other words, we have escaped from the complicated mixed use of filtration and "double gradations" on one graded ring. We are still unable to give a "trivial" proof of [9, Theorem 5.9] in the general case without using the "double gradations."

\section{INVERTIBLE IDEALS}

Let $R$ be a ring and $I$ an ideal of $R$. Consider an overring $T$ of $R$ such that there is an $R$-bisubmodule $J$ of $T$ such that $I J=J I=R$. Then $I$ is said to be invertible (in $T$ ), and one writes $J=I^{-1}$. To such an $I$, there are two associated graded rings, i.e., $R(I)=\bigoplus_{n>0} I^{n} X^{n} \subset R[X]$, the negative part of the Rees ring of $R$ with respect to the $I$-adic filtration on $R ; \check{R}(I)=\bigoplus_{n \in \mathbb{Z}} I^{n} X^{n} \subset T\left[X, X^{-1}\right]$, the Rees ring of the filtered overring $T(I)=\bigcup_{n \in \mathbb{Z}} I^{n} \subset T$ of $R . \check{R}(I)$ is called the generalized Rees ring of $R$ with respect to $I$. These rings play an important part in [5, 4]. Note that $\check{R}(I)$ has gradation, i.e., $\check{R}(I)_{n}=I^{-n} X^{-n}, n \in \mathbb{Z}$, in particular $\check{R}(I)_{0}=R$ and $R \subset I^{-1}$. One sees that $X^{-1} \in \check{R}(I)_{1}$ is central homogeneous of degree 1 in $\check{R}(I)$. Put $Y=X^{-1}$. Then in view of Proposition 3.2 we have the following

5.1. Observations. Consider the filtered ring $A=\check{R}(I) /(1-Y) \check{R}(I)$ defined as in $\S 3$ with filtration $F A=\left\{F_{n} A=\left(\check{R}(I)_{n}+(1-Y) \check{R}(I)\right) /(1-Y) \check{R}(I)\right.$, $n \in \mathbb{Z}\}$ and the localization $\check{R}(I)_{(Y)}$ of $\check{R}(I)$ at the multiplicative closed set $\left\{1, Y, Y^{2}, \ldots\right\}$. Then

(1) $A \cong T(I) \cong\left(\check{R}(I)_{(Y)}\right)_{0}$.

(2) $G(A) \cong \check{R}(I) / Y \check{R}(I)$ where $G(A)_{0} \cong R / I$.

(3) $G(A)^{-} \cong \bigoplus_{n \geq 0} I^{n} / I^{n+1}=G_{I}(R)$, where $G_{I}(R)$ denotes the associated graded ring of $R$ with respect to the $I$-adic filtration on $R$.

(4) $I \subset J(R)$ if and only if $Y \in J^{g}(\check{R}(I))$ if and only if $F_{-1} A \subset J\left(F_{0} A\right)$.

5.2. Theorem. Let $R$ be a ring and $I$ an invertible ideal of $R$. With the above notation, the following are equivalent :

(1) $R$ is Noetherian (or equivalently $\check{R}(I)$ is Noetherian), and $I \subset J(R)$.

(2) The I-adic filtration on $R$ is Zariskian.

(3) $R / I$ is Noetherian and $\widehat{A}$ is faithfully flat as an A-module (this condition is equivalent to saying that $F A$ is Zariskian).

Proof. By Observation 5.1 and the definition of Zariskian filtration, the equivalence is obvious.

5.3. Theorem. Let $R$ be $a$ ring and $I$ an invertible ideal of $R$. Suppose that $R$ is Noetherian and $I \subset J(R)$. Then

(1) if $\operatorname{gl} \operatorname{dim}(R / I)<\infty$, then

(a) gr. gl. $\operatorname{dim} \check{R}(I)=1+$ gr.gl. $\operatorname{dim} G(A)=1+$ gl. $\operatorname{dim} R / I$;

(b) gl. $\operatorname{dim} R=1+$ gl. $\operatorname{dim} R / I$; 
(c) $\mathrm{gl} \cdot \operatorname{dim} \check{R}(I)=1+$ gl. $\operatorname{dim} G(A) \leq 2+$ gl. $\operatorname{dim} R / I, \operatorname{gl} \cdot \operatorname{dim} \widetilde{R}=1+$ gl. $\operatorname{dim} G_{I}(R) \leq 2+$ gl. $\operatorname{dim} R / I$ where $\widetilde{R}$ resp. $G_{I}(R)$ is the Rees ring resp. associated graded ring of $R$ with respect to the I-adic filtration on $R$.

(2) If $R / I$ is Auslander regular then $R$ and $\check{R}(I)$ are Auslander regular.

Proof. By the assumptions on $R$ and $I$, we can use Theorem 5.2, Observation 5.1 , the properties of a strongly graded ring, and [10,9] to prove the theorem.

5.4. Theorem. Let $R$ be a ring and $I$ an invertible ideal of $R$. Suppose that $R($ or $\check{R}(I))$ is Noetherian. Then

(1)

$$
\text { gl. } \begin{aligned}
\operatorname{dim} \check{R}(I) & \leq \max \{1+\text { gl. } \operatorname{dim} G(A), 1+\text { gl. } \operatorname{dim} A\} \\
& \leq \max \{2+\text { gl. } \operatorname{dim}(R / I), 1+\text { gl. } \operatorname{dim} A\} .
\end{aligned}
$$

If $\operatorname{gl} \operatorname{dim}(R / I)<\infty$ then the first equality holds.

(2) If $R / I$ and $A$ are Auslander regular then $R$ and $\check{R}(I)$ are Auslander regular.

Proof. Use Observation 5.1, Theorem 4.4, and the results for nonZariskian ring in [7].

Now, let us consider the graded invertible ideal. Again, we need some more technical preparation work.

5.5. New strongly graded structure over a strongly graded ring. Let $R$ be any $\mathbb{Z}$ graded ring, say $R=\bigoplus_{n \in \mathbb{Z}} R_{n}$. Consider the graded subring $R^{+}=\bigoplus_{n \geq 0} R_{n}=$ $\bigoplus_{n \geq 0}\left(R_{1}\right)^{n}$, where we have put $\left(R_{1}\right)^{0}=R_{0}$.

Let $U=R \bigotimes_{R_{0}} R^{+}$, i.e., we are looking at $R^{+}$as an $R_{0}$-bimodule and constructing the strongly graded $R$-module as usual. Then

$$
U_{k}=R_{k} \bigotimes_{R_{0}} R^{+} \cong \bigoplus_{n \geq 0}\left(R_{k} \bigotimes_{R_{0}} R_{n}\right) \cong \bigoplus_{n \geq 0} R_{k+n} .
$$

This shows that each $U_{k}, k \in \mathbb{Z}$ is a subgroup of $R$. So by passing to the multiplication on $R$, we obtain a strongly graded ring structure on $U$ such that

$$
U_{0} \cong R^{+} \text {. }
$$

On the other hand, consider the polynomial ring $R[y]=\bigoplus_{n \geq 0} R y^{n}$. Then since for any $s_{t} \in R_{t}, s_{t} y^{n} \in R_{t} y^{n}=R_{(t-n)+n} y^{n}$, it follows that

$$
R[y]=\bigoplus_{n \geq 0} R y^{n}=\bigoplus_{k \in \mathbb{Z}}\left(\bigoplus_{n \geq 0} R_{k+n} y^{n}\right) \cong \bigoplus_{k \in \mathbb{Z}} U_{k}=U,
$$

and the latter isomorphism is a ring morphism.

5.6. Remark. (1) Similarly, we may obtain the same result for the graded subring $R^{-}=\bigoplus_{n \leq 0} R_{n}$ of $R$.

(2) The construction we made above may be applied to study the strongly graded ring given by the tensor ring structure determined by an element in $\operatorname{Pic}(R)$ of a ring $R$, but this is the task of our forthcoming work. 
5.7. Lemma. Let $R$ be a G-graded ring by a group $G$ and $I$ a graded ideal of $R$. Suppose that I has the Artin-Rees property. Then the following statements are equivalent :

(1) $I$ is contained in the graded Jacobson radical $J^{g}(R)$ of $R$.

(2) For any finitely generated graded $R$-module $M$ and any graded $R$ submodule $N$ of $M, N=\bigcup_{n \geq 1}\left(N+I^{n} M\right)$.

Proof. Note that $\cap\left(N+I^{n} M\right)$ is a graded submodule of $M$; this is a graded version of [NVO13, Chapter D, Proposition 5.7].

5.8. Lemma. Let $R$ be a G-graded ring and I a graded ideal of $R$. Suppose that $R$ is Noetherian and $I$ is an invertible ideal contained in $J^{g}(R)$. Consider the I-adic filtration on $R$. Then

(1) any good filtration on a graded $R$-module is separated.

(2) The Rees ring $\widetilde{R}$ of $R$ associated to the I-adic filtration is Noetherian.

(3) Good filtrations induce good filtrations on R-submodules.

Proof. By the assumptions it follows that $I$ has Artin-Rees property (see [13]) and, hence Lemma 5.7 works properly. The rest of proof is similar to [13, Lemma 5.1].

5.9. Lemma. Let $R$ and $I$ be as in Lemma 5.8. Suppose that $R$ is Noetherian and $I$ is contained in $J^{g}(R)$. Consider the I-adic filtration on $R$. Let $M$ be a finitely generated graded $R$-module with a good filtration $F M$. Then for any $j=0,1, \ldots, \operatorname{Ext}_{R}^{j}(M, R)$ is a finitely-generated graded $R$-module, and there exists a good filtration on $\operatorname{Ext}_{R}^{j}(M, R)$ such that $G\left(\operatorname{Ext}_{R}^{j}(M, R)\right)$ is isomorphic to a subquotient of $\operatorname{Ext}_{G(R)}^{j}(G(M), G(R))$.

Proof. By using Lemma 5.8 this is a graded version of [10, Theorem 4.7].

5.10. Theorem. Let $R$ be a $\mathbb{Z}$-graded ring. Suppose that $R$ is left and right Noetherian and $I$ is an invertible graded ideal of $R$ contained in $J^{g}(R)$. If $R / I$ is Gorenstein, then $R$ is Gorenstein.

Proof. Consider the $I$-adic filtration on $R$. Then by Observation 5.1 the associated graded ring $G_{I}(R)$ of $R$ is isomorphic to the negative part $G(A)^{-}$of $G(A)$ as mentioned in Observation 5.1(3). Since $G(A)$ is strongly graded with $R / I$ as the part of degree zero, it follows that $G(A)$ is graded Gorenstein. But then by using the construction given in foregoing 5.5 for $G(A)$ and [LIVO2], we may derive that $G(A)^{-}$and hence $G_{I}(R)$ is Gorenstein. Now use Lemma 5.8 and Lemma 5.9. We may prove that $R$ has finite graded injective dimension, and hence by Example (ii) of $\S 3 . R$ has finite injective dimension. Then in view of Lemma 3.7 again a similar argument as in the proof of [9, Proposition 5.3] shows that every finitely-generated graded $R$-module satisfies the Auslander condition (A3) (see §4). Finally, by a similar proof as in [9] again we may conclude that every finitely-generated $R$-module satisfies (A3).

5.11. Corollary. Let $R$ and $I$ be as in Theorem 5.10. Suppose that $R$ has finite global dimension. If every finitely generated $R / I$-module satisfies (A3), then $R$ and $\check{R}(I)$ are Auslander regular. In particular, if $R$ and $I$ are as in Theorem 5.3 and $R / I$ is Auslander regular, then the Rees ring $\widetilde{R}$ of $R$ with respect to the I-adic filtration is Auslander regular. 


\section{REFERENCES}

1. M. J. Asensio, M. Van den Bergh, and F. Van Oystaeyen, A new algebraic approach to microlocalization of filtered rings, Trans. Amer. Math. Soc. 316, (1989) 537-555.

2. A Borel, Algebraic D-modules, Academic Press, London and New York, 1987.

3. W. Bruns and U. Vetter, Determinantal rings, Lecture Notes in Math., vol. 1327, SpringerVerlag, 1988

4. L. Le Bruyn, M. Van den Bergh, and F. Van Oystaeyen, Graded orders, Birkhäuser, Boston and Basel, 1988.

5. L. Le Bruyn and F. Van Oystaeyen, Generalized Rees rings satisfying polynomial identities, J. Algebra 83 (1983), 404-436.

6. Hui-Shi Li, Non-commutative Zariskian iings, thesis, Antwerp, 1990.

7. Hui-Shi Li, M. Van den Bergh, and F. Van Oystaeyen, The global dimension and regularity of non-Zariskian rings, Comm. Algebra (to appear).

8. Hui-Shi Li and F. Van Oystaeyen, Filtrations on simple Artinian rings, J. Algebra 132, 1990, 361-376.

9. $\ldots$ Global dimension and Auslander regularity of graded rings and Rees rings, Bull. Soc. Math. Belg. XLIII, 1991, pp. 59-87.

10. __ Zariskian filtrations, Comm. Algebra 17 (1989), 2945-2970.

11. __ Zariskian Filtrations, Monograph, (to appear).

12. Hui-Shi Li, F. Van Oystaeyen, and E. Wexler-Kreindler, Zariskian rings and flatness of completion, J. Algebra, 138, 2, 1991, 327-339.

13. C. Năstăsescu and F. Van Oystaeyen, The dimensions of ring theory, Reidel, Dordrecht, Holland, 1987.

14. __ Graded rings theory, Math. Library, vol. 28, North Holland, Amsterdam, 1982.

15. D. G. Northcott, An introduction to homological algebra, Cambridge Univ. Press, London and New York, 1960.

16. J. J. Rotman, An introduction to homological algebra, New York, 1979.

17. M. Van den Bergh and F. Van Oystaeyen, Lifting maximal orders, Comm. Algebra 17 (1989), 341-349.

18. O. Zariski and P. Samuel, Commutative algebra, Vol. I and II, Van Nostrand, 1958; New Printing, Springer, New York, 1960.

Department of Mathematics, ShaAnXi Normal University, Xian, People's Republic of CHINA

Department of Mathematics and Computer Science, University of ANTWerp, UIA, 2610 WILRIJK, BELGIUM 